\title{
Thrombosis and Bleeding in Philadelphia Negative Myeloproliferative Neoplasia: Incidences and Risk Factors
}

\author{
Mufide Okay ${ }^{1, *}$, [MD] \\ ORCID: 0000-0001-5317-0597 \\ Umit Yavuz Malkan ${ }^{2}$, [MD] \\ ORCID: 0000-0001-5444-4895 \\ Ertugrul Cagri Bolek ${ }^{3}$, [MD] \\ ORCID: 0000-0003-3886-2813 \\ Nilgun Sayinalp ${ }^{1}$, [MD] \\ ORCID: 0000-0002-4782-896X \\ Yahya Buyukasik ${ }^{1}$, [MD] \\ ORCID: 0000-0002-4764-2348 \\ Ibrahim Haznedaroglu ${ }^{1}$, [MD] \\ ORCID: 0000-0001-8028-9462
}

1, Hacettepe University Medical School, Department of Hematology, Ankara, TURKEY.

2,University of Health Sciences, Dışkapı Yıldırım Beyazıt Training and Research Hospital, Department of Hematology, Ankara, TURKEY

3,Hacettepe University Medical School, Department of Internal Medicine, Ankara, TURKEY

*Corresponding Author: Mufide Okay, MD

Hacettepe University Medical School

Department of Hematology

06100, Ankara, Turkey

Phone: +903123051536

Fax: +903123051714

e-mail: mufideokay87@gmail.com

\section{we ABSTRACT Cem}

Objective: Thrombohemorrhagic events developing in the Philadelphia negative myeloproliferative neoplasms may worsen the clinical course of the disease and increase the morbidity and mortality. The aim of this study is to assess critical risk factors of the hemostatic complications in myeloproliferative neoplasms.

Materials and Methods: The patients who were diagnosed as myeloproliferative neoplasm based on world health organization 2016 criteria are included into the study. The categorical and continuous data were compared with Chi-square and independent sample t-test. The statistical significance threshold was considered as $\mathrm{p}<0.05$.

Results: 181 patients were evaluated in this study. The number of the patients with polycythemia vera, essential thrombocytosis and primary myelofibrosis were 89, 50 and 42 respectively. At the time of diagnosis, the median age was 54 years. There were 50 patients who had thrombosis at the time of diagnosis, and 22 patients have developed thrombosis during the follow-up. Venous thrombosis is more frequent than arterial thrombosis. The smoking habit was statistically higher in the patient with myeloproliferative neoplasm who developed thrombosis $(\mathrm{p}=0.006)$. The most frequent hemorrhagic complication was gastrointestinal bleeding. Platelet levels of patients with complications during the course of myeloproliferative neoplasm were found to be lower than those without complications ( $\mathrm{p}<0.001)$.

Conclusion: The hemostatic complication rates due to myeloproliferative neoplasm were $39 \%$ thrombosis and $13 \%$ bleeding. While the cigarette usage was significantly higher in the thrombosis group, advanced age, male sex, types of myeloproliferative neoplasm, JAK-2 positivity were similar between the groups. Further experimental and clinical studies should focus on the pathobiology and proper clinical management of the myeloproliferative neoplasm patients with hemostatic complications.

Keywords: Myeloproliferative disorders, risk factors, thrombosis.

\section{INTRODUCTION}

Myeloproliferative neoplasms (MPN), namely essential thrombocytosis $(E T)$, polycythemia vera $(\mathrm{PV})$, primary myelofibrosis (PMF), are the clonal neoplastic diseases characterized by neoplastic production of bone marrow hematopoiesis. Thrombosis and bleeding are the major complications that increase the rates of mortality and morbidity [1]. Thromboses of MPNs could be observed in both 
arterial and venous systems [2]. Atypical thrombotic regions (splenic vascular bed, splanchnic vein thrombosis, Budd-Chiari syndrome, cerebral sinus venous thrombosis) rather than in classical ones (coronary, cerebral artery thrombosis, deep vein thrombosis and pulmonary embolism) should alert the clinician regarding diagnosis of MPN. The possible causes of thrombosis in MPN include vascular disturbance, platelet activation, endothelial damage, microparticle-induced coagulation, age and previous thrombosis. Platelet activation leading to platelet leukocyte adherence, endothelial activation and consequent initialization of the coagulation could also play a significant role in the pathogenesis of MPN thrombosis [3].

In the course of MPN, besides life-threatening bleedings such as the variceal bleeding and systemic hemorrhage in presence of portal hypertension, also the minor bleedings including ecchymosis, gingival bleeding, menorrhagia and epistaxis may be seen [3]. Various risk factors have been defined in MPN-associated bleeding. Thrombocytopenia, platelet dysfunction, acquired von Willebrand syndrome (aVWS), older age, duration of illness and previous history of bleeding are the well described risk factors [3].

The aim of this study is to assess critical risk factors of the hemostatic complications in MPN

\section{MATERIALS and METHODS}

We retrospectively analyzed 6582 patients who had a cytosis change in their hematological blood values. According to the world health organization (WHO) criteria, it was reached to 241 patients who had been diagnosed as MPN [4]. 60 patients with very insufficient information for the study were excluded from the analyses. A total of 181 patients diagnosed with MPN in our center between January 1994 and December 2017 were included into the study. A written consent form was signed by the patients at the time of admission. Patients' information was reached via using Nucleus and Core databases. The conduct of this study was approved by the Institutional Review Board (GO 17/926).

For each of the patients, the following information was recorded: demographic data, the presence of microvascular disturbances (erythromelalgia) or constitutional symptoms (pruritus, fatigue), diagnosis (PV, ET, PMF), laboratory values, results of the laboratory investigation for thrombophilia, mutational profile, concomitant diseases (cancer), the presence of cardiovascular risk factors (smoking, hypertension, dyslipidemia, diabetes), clinical treatment modalities, history of thrombosis and bleeding complications, causes of death.

Major thrombotic events were analyzed as arterial (ischemic stroke, acute myocardial infarction) and venous (deep vein thrombosis with or without pulmonary embolism, splanchnic vein thrombosis) systems. Among all of the thrombohemorrhagic events recorded in the patient files before and/or after MPN diagnosis, only those confirmed through clinical evaluation, laboratory, and/or imaging modalities were considered for this assessment. Hemorrhagic complications were classified as the major (gastrointestinal and cerebrovascular bleedings requiring hospitalization) and minor (skin, gingival, genitourinary system hemorrhages) bleedings.

The patients were divided into two groups according to having or not having thrombosis and bleeding at the time of diagnosis and follow-up. The differences between the two groups in terms of MPN diagnoses, age, gender, cardiovascular risk factors, and laboratory data and whether there is the previous history of thrombosis and bleeding were identified.

Statistical Analyses

Categorical and continuous data were compared with Chi-square and independent sample t-test. For comparison of more than two group means, the one-way analysis of variance (ANOVA) was used in data which showed normal distribution pattern and Kruskal-Wallis method was used in data which did not show normal pattern. IBM SPSS Statistics for Windows, Version 20.0. (Armonk, NY: IBM Corp.) software were used for the statistical analysis. Data were given as median (minimum-maximum) unless indicated. The statistical significance threshold was considered as $p<0.05$.

\section{RESULTS}

A total of 181 patients were evaluated. The number of the patients with PV, ET, and PMF were 89, 50 and 42 , respectively. At the time of diagnosis, the median age was 54 years (16-89). There were 72 patients who had thrombosis (39.7\%). Fifty of them had at the time of diagnosis and 22 patients had developed thrombosis during follow-up. The number of the patients with venous thrombosis was higher than that of the patients with arterial thrombosis. Portal vein thrombosis ( 14 patients) and Budd-Chiari syndrome (13 patients) were the most frequent 
cases of venous thrombosis. Other venous thrombosis events include splanchnic vein, deep vein, dural sinus, isolated mesenteric thrombosis in 5, 2, 1, 1 patient, respectively. Ischemic stroke (4 patients) and splenic artery-mesenteric artery thrombosis (4 patients) were the most frequent cases of arterial thrombosis. The other arterial thrombosis cases were 2 patients with acute myocardial infarction, 1 patient with digital artery thrombosis, 1 patient with brachial artery thrombosis, 1 patient with aorta abdominal thrombosis and 1 patient with femoral artery thrombosis. Following findings were detected in the thrombophilia panel of 47 patients: various polymorphisms were detected in 26 patients,
MTHFR677 gene polymorphism in 5 patients and heterozygous Factor $V$ Leiden mutation was detected in 5 patients.

There were 5 patients who were diagnosed with MPN with bleeding complication and 19 patients with bleeding complications at follow-up. The most frequent complication was gastrointestinal bleeding. Of the bleeding events, 14 (7.7\%) were gastrointestinal bleedings and $1(0.6 \%)$ was cerebrovascular bleeding. In addition, 9 (5\%) patients had minor bleeding such as skin, gingival, and genitourinary bleedings. Other demographic characteristics of the patients are given in Table 1.

Table 1. Patients' characteristics in the study group of MPN.

\begin{tabular}{|c|c|}
\hline Characteristics & $\mathrm{N} \%$ \\
\hline Age (median, range) & $54(16-89)$ \\
\hline Female/Male & $83 / 98$ \\
\hline \multicolumn{2}{|l|}{ Symptoms } \\
\hline Erythromelalgia & 25/179 (13.9\%) \\
\hline Pruritus & $29 / 150(19.3 \%)$ \\
\hline \multicolumn{2}{|l|}{ Cardiovascular risk factors } \\
\hline Smoking & $52 / 180(28.7 \%)$ \\
\hline Diabetes mellitus & $33 / 181(18.2 \%)$ \\
\hline Hypertension & $68 / 181(37.6 \%)$ \\
\hline Dyslipidaemia & $34 / 181(18.8 \%)$ \\
\hline History of thrombosis & 19/181 (10.5\%) \\
\hline History of bleeding & $12 / 181(6.6 \%)$ \\
\hline \multicolumn{2}{|l|}{ MPN diagnosis } \\
\hline PV & 89 (49.2\%) \\
\hline ET & $50(27.6 \%)$ \\
\hline PMF & $42(23.2 \%)$ \\
\hline Presence of thrombophilia & $26 / 181(14.4 \%)$ \\
\hline \multicolumn{2}{|l|}{ Mutation profile } \\
\hline JAK2 mutation & $115 / 144(79.8 \%)$ \\
\hline CALR mutation & $2 / 7(28.5 \%)$ \\
\hline MPL mutation & $1 / 7(14.2 \%)$ \\
\hline Thrombosis at the time of diagnosis & $50 / 181(27.6 \%)$ \\
\hline Bleeding at the time of diagnosis & 5/181 (2.8\%) \\
\hline
\end{tabular}


No difference was detected among the groups according to age and gender when the groups were compared according to having/not having thrombosis during being diagnosed or at follow-up ( $p=0.34$ and $p=0.76$ ). When the laboratory values were compared; the median hemoglobin value was $14.1 \mathrm{gr} / \mathrm{dL}$ in the thrombosis group and $13.3 \mathrm{gr} / \mathrm{dL}$ in the non-thrombosis group $(p=0.09)$. While no difference was observed in terms of white blood cells, platelet values were found to be lower in the thrombosis group $(p<0.001)$. Moreover, there was no difference in terms of JAK2 mutation positivity among the studied groups.
The smoking rate was statistically higher in the patients diagnosed with or without thrombosis $(p=0.006)$. No association with other cardiovascular risk factors was found as well (Table 2).

No difference was detected among the groups according to the age and gender when the groups were compared according to having/not having bleeding during being diagnosed or at follow-up period $(p=0.49$ and $\mathrm{p}=0.99$ ). No statistically significant difference between the groups was detected when the groups were compared according to the laboratory values and cardiovascular risk factors (Table 2).

Table 2. The differences between the patients with/without thrombosis and bleeding in MPN.

\begin{tabular}{|c|c|c|c|c|c|c|}
\hline Characteristics & $\begin{array}{l}\text { Thrombosis } \\
\qquad(\mathrm{n}=72)\end{array}$ & $\begin{array}{c}\text { No Thrombosis } \\
(\mathrm{n}=109)\end{array}$ & $\mathrm{p}$ & $\begin{array}{l}\text { Bleeding } \\
(\mathrm{n}=24)\end{array}$ & $\begin{array}{l}\text { No bleeding } \\
\quad(n=157)\end{array}$ & $\mathrm{p}$ \\
\hline Age & 52 & 54 & 0.34 & 55 & 53 & 0.49 \\
\hline Female/Male & $34 / 38$ & $49 / 60$ & 0.76 & $11 / 13$ & $72 / 85$ & 0.99 \\
\hline Diagnosis & & & & & & \\
\hline PV & 43 & 46 & \multirow{3}{*}{0.041} & 11 & 78 & \multirow{3}{*}{0.93} \\
\hline ET & 18 & 32 & & 7 & 43 & \\
\hline PMF & 11 & 31 & & 6 & 36 & \\
\hline Hemoglobine (median) (gr/dL) & 14.1 & 13.3 & 0.09 & 13.0 & 13.8 & 0.31 \\
\hline $\begin{array}{l}\text { Leukocyte count (median) } \\
\left(\times 10^{3}\right)\end{array}$ & 13.3 & 12.9 & 0.81 & 13.2 & 13.0 & 0.96 \\
\hline Platelet count (median) $\left(\times 10^{3)}\right.$ & 242 & 529 & 0.00 & 298 & 433 & 0.12 \\
\hline JAK-2 & & & & & & \\
\hline Positive & 52 & 63 & \multirow{2}{*}{0.11} & 16 & 99 & 0.51 \\
\hline Negative & 10 & 19 & & 2 & 27 & \\
\hline $\mathrm{HT}^{*}$ & 24 & 44 & 0.33 & 10 & 58 & 0.65 \\
\hline $\mathrm{DM}+$ & 9 & 24 & 0.1 & 4 & 29 & 0.83 \\
\hline Smoking & 29 & 23 & 0.006 & 9 & 43 & 0.31 \\
\hline
\end{tabular}

*HT: hypertension, † DM: diabetes mellitus.

We analyzed MPN patients based on the following groups; the clinical course of MPN were lower than those without a) patients who had only bleeding b) patients who had only complications [183×103 $(131 \times 103-490 \times 103)$ vs. $563 \times 103$ thrombosis $c)$ patients who had both hemorrhagic compli- $(11 \times 103-2282 \times 103)] \quad(p<0.001)$. Moreover, a statistically cations and thrombosis at the follow-up or during being di- significant difference was observed between the groups agnosed; no difference was observed between the groups in terms of cigarette usage, which was higher in the in terms of age, gender, and MPN diagnoses. Platelet lev- thrombosis groups $(p=0.02)$ (Table 3

els of the patients with hemostatic complications during 
Table 3. Comparison of risk factors of patients according to complication groups.

\begin{tabular}{|c|c|c|c|c|c|}
\hline & $\begin{array}{c}\text { Patients who } \\
\text { had only } \\
\text { bleeding }(n=17\end{array}$ & $\begin{array}{l}\text { Patients who had } \\
\text { only thrombosis } \\
\qquad(n=65)\end{array}$ & $\begin{array}{l}\text { Bleeding } \\
\text { plus Thrombosis } \\
\qquad(n=7)\end{array}$ & $\begin{array}{l}\text { Patients } \\
\text { without complication } \\
\qquad(\mathrm{n}=92)\end{array}$ & $p$ \\
\hline Age & 57 & 52 & 49 & 53 & 0.57 \\
\hline Female/Male & $8 / 9$ & $31 / 34$ & $3 / 4$ & $41 / 51$ & 0.98 \\
\hline \multicolumn{6}{|l|}{ Diagnosis } \\
\hline PV & 7 & 39 & 4 & 39 & \\
\hline ET & 5 & 16 & 2 & 27 & 0.39 \\
\hline PMF & 5 & 10 & 1 & 26 & \\
\hline $\mathrm{Hb}(\mathrm{gr} / \mathrm{dL})$ & 13 & 14.3 & 13 & 13.4 & 0.27 \\
\hline Leukocyte & 12.3 & 13.1 & 15.2 & 13 & 0.96 \\
\hline$\left(x 10^{3}\right)$ & 345 & 248 & 183 & 563 & 0.00 \\
\hline \multicolumn{6}{|l|}{ Platelets } \\
\hline \multicolumn{6}{|l|}{$\left(x 10^{3}\right)$} \\
\hline \multicolumn{6}{|l|}{ JAK-2 } \\
\hline Positive & 11 & 47 & 5 & 52 & 0.36 \\
\hline Negative & 2 & 10 & 0 & 17 & \\
\hline $\mathrm{HT}^{*}$ & 8 & 22 & 2 & 36 & 0.71 \\
\hline DM† & 4 & 9 & 0 & 20 & 0.32 \\
\hline Smoking & 5 & 25 & 4 & 18 & 0.02 \\
\hline
\end{tabular}

*HT: hypertension, † DM: diabetes mellitus.

Forty-eight patients were accepted as documented dropouts during the follow-up period because of death. The documented causes of deaths; 1 patient was dead due to pneumonia-induced sepsis, 4 patients due to the massive gastrointestinal bleeding, 2 patients were died following chemotherapy of leukemic transformation, 1 patient due to the liver malignant tumor and liver failure during follow-up, 1 patient due to liver failure considered as being secondary to massive thrombosis and 1 patient was died due to viral encephalitis. The remaining causes of mortality were failed to be identified. 


\section{DISCUSSION}

Most MPN patients experience hemostatic complications such as bleeding or thrombosis one or more times. Thrombotic complications are observed in more than $30 \%$ of patients with MPN [3]. The incidence rate of bleeding in PV, PMF, and ET patients is $3-8.1 \%, 12 \%$, and $3-18 \%$ respectively $[5,6]$. Bleeding and thrombosis are the major causes of morbidity and mortality in MPN. Since the mechanism of bleeding or thrombosis could not be fully elucidated, various hemostatic risk factors have been defined. In this study, we determined the frequency and the risk factors of hemorrhagic and thrombosis events in PV, ET, PMF patients who were admitted to our clinic and followed up.

In our MPN cohort, thrombosis and bleeding as the major complications were comparable to the previous published literature (39\% thrombosis, $13 \%$ bleeding). It has been suggested that being older than 60 years of age and having previous thrombosis history are the main factors predicting the risk of thrombosis [7]. In our study, no significant difference in terms of age was detected among the patients with and without thrombosis. However, in patients who had thrombosis, the previous history of thrombosis was higher compatible with the literature $(p=0.028)$. When we divided the MPN groups according to the bleeding or thrombosis occurred / not occurred at the time of diagnosis or follow-up, it was observed that having previous thrombosis history also increased the risk of thrombosis $(p=0.055)$ (data not shown). There was a speculation that male gender can predict VTE for ET [3]. However, in our MPN cohort, no significant difference in terms of gender was detected among the studied groups.

The conducted studies have shown that cardiovascular risk factors (smoking, hypertension $(\mathrm{HT})$, diabetes mellitus (DM)) and classical venous thromboembolism (VTE) risk factors (major surgery, oral contraceptive use, pregnancy) increase the risk of thrombosis also in patients with MPN [8-11]. According to the well-established risk factors, only cigarette usage is significant in the thrombosis arm and the other risk factors (DM, HT) are not significant. In addition, previous studies have suggested that having MPN with thrombophilia (such as MTHFR C677T polymorphism) could increase the risk of thrombosis [12]. In our study, although the mutation panel could not be evaluated in all patients due to technical reasons, no difference between the groups was detected.
Leukocytosis may increase the risk of myocardial infarction in PV and ET patients [13]. In this study, no relation was found between the leukocyte count and thrombosis at the follow-up or at the moment of diagnosis in 89 patients with PV and 50 patients with $E T$ ( $p=0.74$ and $p=0.82$, respectively). In the literature, JAK-2 V617F mutation has been found to be a risk factor for thrombotic complications [3]. In the present study, there was no difference in terms of JAK-2 positivity among the groups. Unlike JAK-2 mutation, MPL and CAL-R mutations have been shown to be not correlated with thrombosis in the literature [1]. In our study, it was not possible to comment on this critical issue since the total number of MPN patients examined was only seven.

In a previous study, it has been indicated that splenomegaly was a risk factor for thrombosis [3]. There are published studies suggesting that the spleen size increases the risk of thrombosis. In the present study, the spleen size has been found to be $158 \mathrm{~mm}$ in patients with thrombotic complications that has developed somehow, and there was no significant difference in comparison to the group that has never had thrombosis $(p=0.97)$.

Thrombocytopenia, older age, previous history of bleeding are the risk factors that may be effective on bleeding complications [3]. The presence of thrombocytosis has also been indicated to be a risk factor for bleeding [10]. There are also studies indicating that high platelet count and patient age are not risk factors for bleeding in MPN patients. Also, there was no correlation between thrombocytosis and increased risk of thrombosis in ET patients [7]. In another study, it has been shown that a platelet counts of $>450 \times 109 / L$ was a risk factor for thrombosis in MF patients [1]. Whereas, in our study, there was no correlation between thrombocyte count and thrombotic event in ET patients. In our study, there was no difference in terms of platelet counts in all as the MPN groups when the groups with and without bleeding were compared. However, when all complications were compared, there was a difference between the groups in terms of platelet count. The striking finding in our present study is that MPN patients with relatively low platelet numbers are more prone to hemostatic complication development in comparison to MPN with extreme thrombocytosis $(p<0.001)$ (Table 3$)$. This contrary finding which seems as a paradox may be 
explained by consumption of thrombocytes due to thrombosis, when we consider that the time of performing thrombocyte count was just after the event. Particularly in MPN patients with both bleeding and thrombotic complications at the same time had lower platelet counts when compared to other sub-study groups. Likewise; the MPN patients with thrombosis only had lower median thrombocyte levels in comparison to other hemostatic complication groups. As expected from those data; MPN patients who had only bleeding had lower platelets than those without hemostatic complications (Table 3).

In a previous study, the presence of bleeding history and leukocytosis have been found to be the risk factors for bleeding in patients with PMF [6]. Whereas, in patients only diagnosed as PMF, the platelet count has been found to be lower in the group with bleeding complication $(p=0.01)$. However, no difference has been found in terms of leukocytosis and bleeding history in our MPN cohort.

To recognize the risk factors for bleeding and/or thrombosis in patients with MPH serve us to eliminate or at least to account them in the management of the patients. This would be beneficial in order to improve the clinical course of those patients and manage them properly. For that reason, further experimental and clinical studies should focus on the pathobiology, risk factors and management of the MPN patients with hemostatic complications, namely thrombosis and bleeding.

\section{ACKNOWLEDGEMENTS}

This research received no specific grant from any funding

agency in the public, commercial, or not-for-profit sectors.

M.O. and E.C.B. contributed to data collection, M.O. and I.C.H contributed to writing, U.Y.M. contributed to statistics, N.S., Y.B. and I.C.H. contributed to proof the paper.

\section{CONFLICT Of INTEREST STATEMENT}

The authors declare that there is no conflict of interest.
[1] Martin K. Risk Factors for and Management of MPNAssociated Bleeding and Thrombosis. Current hematologic malignancy reports 2017;12:5389-96.

[2] Baş B, Köksal A, Özatll D, Haznedaroglu I, Koşar A, Büyükaşik Y, Ozcebe $O$, Dündar $S$. Thrombosis and hemorrhage in chronic myeloproliferative disorders. Clinical and Applied Thrombosis/Hemostasis 1999;5:4282-4.

[3] Kaifie A, Kirschner $M$, Wolf $D$, Maintz $C$, Hänel $M$, Gattermann N, Gökkurt E, Platzbecker U, Hollburg W, Göthert J. Bleeding, thrombosis, and anticoagulation in myeloproliferative neoplasms (MPN): analysis from the German SAL-MPN-registry. Journal of hematology \& oncology 2016;9:118.

[4] Norris D, Stone J. WHO classification of tumours of haematopoietic and lymphoid tissues. Geneva: WHO 2008.

[5] Papadakis E, Hoffman R, Brenner B. Thrombohemorrhagic complications of myeloproliferative disorders. Blood reviews 2010;24:6227-32.

[6] Finazzi G, Carobbio A, Thiele J, Passamonti F, Rumi E, Ruggeri M, Rodeghiero F, Randi ML, Bertozzi I, Vannucchi AM. Incidence and risk factors for bleeding in 1104 patients with essential thrombocythemia or prefibrotic myelofibrosis diagnosed according to the 2008 WHO criteria. Leukemia 2012;26:4716.

[7] Wehmeier A, Daum I, Jamin H, Schneider W. Incidence and clinical risk factors for bleeding and thrombotic complications in myeloproliferative disorders. Annals of hematology $1991 ; 63: 2101-6$.
[8] Carobbio A, Thiele J, Passamonti F, Rumi E, Ruggeri M, Rodeghiero F, Randi ML, Bertozzi I, Vannucchi AM, Antonioli E. Risk factors for arterial and venous thrombosis in WHOdefined essential thrombocythemia: an international study of 891 patients. Blood 2011:blood-2011-02-339002.

[9] Barbui T, Finazzi G, Carobbio A, Thiele J, Passamonti F, Rumi E, Ruggeri $M$, Rodeghiero F, Randi ML, Bertozzi I. Development and validation of an International Prognostic Score of thrombosis in WHO-Essential Thrombocythemia (IPSET-thrombosis). Blood 2012:blood-2012-07-444067.

[10] Ruggeri M, Rodeghiero F, Tosetto A, Castaman G, Scognamiglio F, Finazzi G, Delaini F, Mico C, Vannucchi AM, Antonioli E. Postsurgery outcomes in patients with polycythemia vera and essential thrombocythemia: a retrospective survey. Blood 2008;111:2666-71.

[11] Gangat N, Wolanskyj AP, Schwager SM, Mesa RA, Tefferi A. Estrogen-based hormone therapy and thrombosis risk in women with essential thrombocythemia. Cancer 2006;106:112406-11.

[12] De Stefano V, Za T, Rossi E, Fiorini A, Ciminello A, Luzzi C, Chiusolo P, Sica S, Leone G. Influence of the JAK2 V617F mutation and inherited thrombophilia on the thrombotic risk among patients with essential thrombocythemia. Haematologica 2009;94(5):733-7.

[13] Tefferi A, Rumi E, Finazzi G, Gisslinger $H$, Vannucchi A, Rodeghiero F, Randi M, Vaidya R, Cazzola M, Rambaldi A. Survival and prognosis among 1545 patients with contemporary polycythemia vera: an international study. 